\title{
Iodine Content Analysis Using Dual-Energy Computed Tomography as a Biomarker of Transitional Cell Carcinoma, an Experience with Separation of the Clotted Blood and Tumorous Tissue
}

\author{
HYNEK MIRKA $^{1,2}$, JAN BAXA $^{1}$, MILAN HORA $^{3}$, ONDREJ HES ${ }^{4}$, ONDREJ TOPOLCAN ${ }^{5}$ and JIRI FERDA ${ }^{1}$ \\ Departments of ${ }^{1}$ Imaging Methods and ${ }^{3}$ Urology, ${ }^{4}$ Sikl's Department of Pathology, and \\ ${ }^{5}$ Central Laboratory for Immunoanalysis, Medical School and Teaching Hospital Pilsen, \\ Charles University in Prague, Pilsen, Czech Republic; \\ ${ }^{2}$ Biomedical Centre, Faculty of Medicine in Pilsen, Charles University in Prague, Pilsen, Czech Republic
}

\begin{abstract}
Background/Aim: The aim of this study was to evaluate the possibility to discriminate the blood clot from tumorous tissue in the assessment of transitional cell carcinoma (TCC), when CT data are used only from singlephase dual-energy CT (DECT). Materials and Methods: A total of 18 patients were included into the retrospective analysis of the prospectively collected data (the average age was 67.3 years, range $=62-79$ years, 12 males, 6 females $)$. DECT was performed in all patients after intravenous administration of the iodinated contrast material. DECT analysis using three-material decomposition algorithm was used to discriminate blood and enhancing tumorous tissue. In all patients, TCC was detected and subsequently kidney surgery or endoscopy was performed within the next two weeks. The findings were compared with DECT results. Results: In our cohort of patients we found 9 TCC of the renal pelvis or ureter and 7 TCC of the urinary bladder. The accuracy of the TCC detection was 94\% (17/18). The presence of coagulated blood within the urinary collecting system together with a tumor was detected in 7 CT examinations. The blood coagulum without a confirmed malignant tumor was found in one case with false positive DECT finding. In other 6 cases bleeding was confirmed during surgery or endoscopy (accuracy 87\%). Conclusion: The dual-energy data analysis enables discrimination of a blood clot from tumorous tissue.
\end{abstract}

Correspondence to: Dr. Hynek Mírka, Department of Radiology, Medical School and Teaching Hospital Pilsen, Charles University in Prague, Alej Svobody 80, 30460 Pilsen, Czech Republic. Tel: +420 377103456, Fax: +420 377103438, e-mail: mirka@fnplzen.cz

Key Words: Dual-energy CT, hematuria, transient cell carcinoma, urinary tract tumors.
It helps in detection of UCC in cases when the excretion potential of the kidneys is decreased due to fulfillment of the collecting system with blood clots.

Transitional cell carcinoma (TCC) is a malignant tumor arising from the transitional epithelial cells lining the urinary tract from the renal calyces to the urethra. TCC is the most common tumor of the renal pelvis. Bladder cancer is diagnosed annually in the western world with increasing incidence. Upper urinary tract TCC is estimated to occur in $5 \%$ of all TCC, TCC of the renal pelvis forms less than $10 \%$ of all renal tumors. Evidence indicates that the frequency of urinary collecting system malignancies is increasing. Massive hematuria occurs in many TCC cases, so the detection of the tumor within the collecting system fulfilled with clotted blood is an important diagnostic goal.

Non-contrast CT still represents the gold standard in cases of suspected bleeding in most institutions, since the CT density of coagulated blood is markedly different from those of the soft tissues urine and calcified tissues. Examinations after intravenous administration of a contrast medium are used in detection of vascular structures and enhanced tissue. Kidney imaging has been supplemented by routine contrastenhanced examinations in the arterial, venous and nephrographic phases for imaging of tumors including vascular kidney supply. In recent years, CT urography has become a significant part of the imaging methods in the assessment of the urinary collecting system and it has replaced intravenous urography $(1,2)$, however the role of CT urography in cases of decreasing excretion of urine is still limiting. If CT examination is performed using a dualsource CT, it is possible to acquire dual-energy data by operating two X-ray sources at different voltages (different kV-settings) (3-7). Dual-energy CT (DECT) improves the 
evaluation of examination when an analysis of different tissue types according to their different X-ray attenuation at lower and higher X-ray energies is used. Several clinical studies have been published on the use of DECT analysis for virtual unenhanced imaging of tissues in the abdominal area, lungs and brain (3-11). The aim of this study was to evaluate the possibility to discriminate the blood clot from tumorous tissue in the assessment of transitional cell carcinoma using CT data from single phase postcontrast dual-energy CT.

\section{Materials and Methods}

In total, 18 patients were included in the retrospective study (the average age was 67.3 years, range $=62-79$ years, 12 males, 6 females). All of them underwent DECT and subsequently received surgery or endoscopy due to the suspected TCC. Informed consent was obtained in all patients. Retrospective evaluation of the patient's image data was approved by the Local Ethic Committee. All examinations were performed using a dual-source CT (Somatom Definition, Siemens Healthineers, Forchheim, Germany) operating in dual-energy data acquisition regime.

All dual-energy abdominal CT, ranging from the dome of the diaphragm to the pelvis floor, were performed using $140 \mathrm{kV}$ tube voltage for measurement system $\mathrm{A}$ and $80 \mathrm{kV}$ tube voltage for measurement system B. Collimation was $2 \times(32 \times 0.6 \mathrm{~mm})$ with simultaneous acquisition of 64-slices by means of a $z$-flying focal spot (double z-sampling). The examinations were performed after application of an iodine contrast medium with a concentration of $400 \mathrm{mg}$ of iodine per $\mathrm{ml}$ (Iomeron 400, Bracco, Milan, Italy) with flow rate of $4 \mathrm{ml} / \mathrm{s}$ followed by flush of $50 \mathrm{ml}$ of saline solution. A power injector system was used for the application of the contrast medium and saline solution flushing (Ohio, Ulrich Medical, Ulm, Germany). CT data acquisition was automatically started with the delay of fifteen seconds after the CTdensity in the distal descending aorta exceeded the threshold of $100 \mathrm{HU}$. In this circulation phase after the intravenous administration of the iodinated contrast agent we achieved optimal enhancement of the vessels, kidney parenchyma and collecting system wall.

For each examination, we reconstructed two image data sets, first at $140 \mathrm{kV}$ and second at $80 \mathrm{kV}$. For estimation of the quality of the examination and further evaluation we reconstructed averaged image series with contribution of $80 \mathrm{kV}$ and $140 \mathrm{kV}$ images with ratio of 0.3 . These averaged $\mathrm{CT}$ images are almost equivalent to standard CT images acquired at a tube voltage of $120 \mathrm{kV}$. A medium-smooth body kernel (B31) was used for all reconstructions.

For the purpose of the study, the $80 \mathrm{kV}$ and $140 \mathrm{kV}$ images were further processed using dual energy evaluation software (Syngo Dual Energy - Liver VNC algorithm; Siemens Healthineers). By means of a three-material decomposition algorithm (4) both virtual unenhanced images and maps of iodine distribution were calculated from the DECT data.

The images were anonymized and further evaluated retrospectively and independently by two radiologists (one 15 years' experience with CT, of which 8 years were with MDCT, second with 14 years' experience with $\mathrm{CT}$, of which 8 years were with MDCT). Both radiologists evaluated the presence, location and extent of coagulated blood and tumorous tissue within the urinary collecting system. The evaluation was recorded by each radiologist in a table and both tables were compared. Finally, the image quality of virtual-unenhanced images was rated using three-point scale: 1 - excellent, when the contrast-to-noise virtual-unenhanced images were comparable with averaged CT images; 2 - sufficient, when images were usable in detection of bleeding, stones or masses; and 3 - insufficient, when the images were insufficient for detection of bleeding, stones and masses.

The findings were compared with the results of investigation of the resected organ or with results of endoscopy performed by an experienced urologist. The histological examinations were performed by a pathologist who was an experienced specialist in genitourinary pathology.

\section{Results}

In our cohort of patients, we found 7 cases of TCC within the renal pelvis (Figure 1), 2 TCC in the distal ureter (Figure 2 ), and 7 TCC of the urinary bladder. The presence of clotted blood within the urinary collecting system was detected in 7 cases. In one patient, only chronic cystitis and no tumorous tissue was identified during cystoscopy.

The virtual unenhanced images showed a higher image noise level. Nevertheless, all locations of coagulated blood were well detectable. In the urinary collecting system, the intra-luminal growth of the tumorous tissue differed from coagula on the iodine distribution map with its detectable iodine uptake. The bleeding in the collecting system was identified due to the increased density compared to the surrounding structures on virtual unenhanced images; the tumorous tissue disappeared within the pelvis or ureter due to the subtraction of the density value added by the iodine respectively.

The blood clots were found twice within the renal pelvis, twice both within ureter and urinary bladder and once in the urinary bladder only. All locations were confirmed except from one that was not found in the pelvis during surgery.

The tumorous lesion was confirmed by surgery or endoscopy in 17 cases. The virtual unenhanced imaging regarding the detection of coagulated blood reached the complete agreement between DECT and endoscopy in $87 \%$ (7 of 8 patients). All tumors were correctly found, so the sensitivity reached $100 \%$. The specificity estimation was biased due to the small number of negative cases, so its value of zero showed low validity and is not reported. Regarding the image quality, 13 sets of virtual unenhanced images were of excellent quality, 4 sufficient, and none insufficient. The average rating was 1.25 , which means that the image quality was slightly deteriorated, but the ability to assess it was not significantly impaired.

The averaged contrast-to-noise ratio between urine and muscle tissue reached 2.25 (ranged $=1.73-3.12$ ) in virtual unenhanced CT images, and 4.32 (ranged=2.92-5.80) in contrast enhanced original CT images respectively.

\section{Discussion}

Unenhanced abdominal imaging belongs within the basic algorithms of the diagnosis of urinary calculi $(1,2,10)$. Unenhanced imaging helps to detect all structures with high 

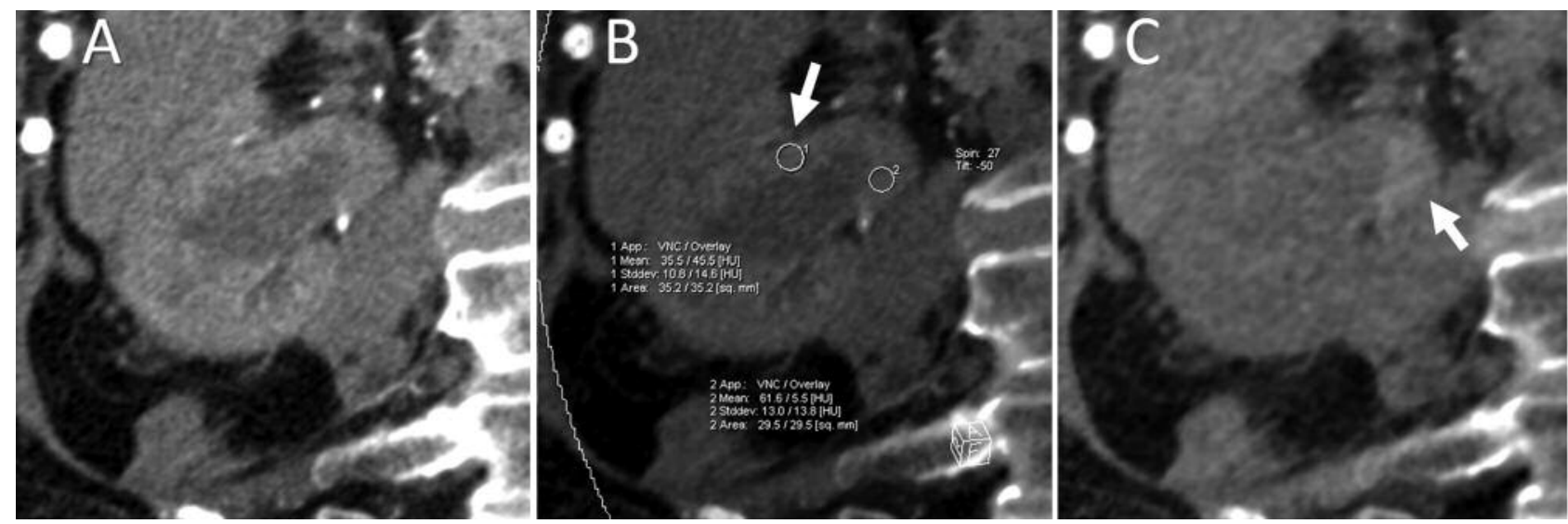

Figure 1. Transition cell carcinoma of the renal pelvis. A: Conventional enhanced image (DECT averaged image). B: DECT iodine map showing an enhancement of tumorous tissue (arrow), no enhancement in coagulated blood. C: DECT virtual unenhanced image showing the hyperdense coagula within pelvis (arrow) and absence of increased in density of tumorous tissue.
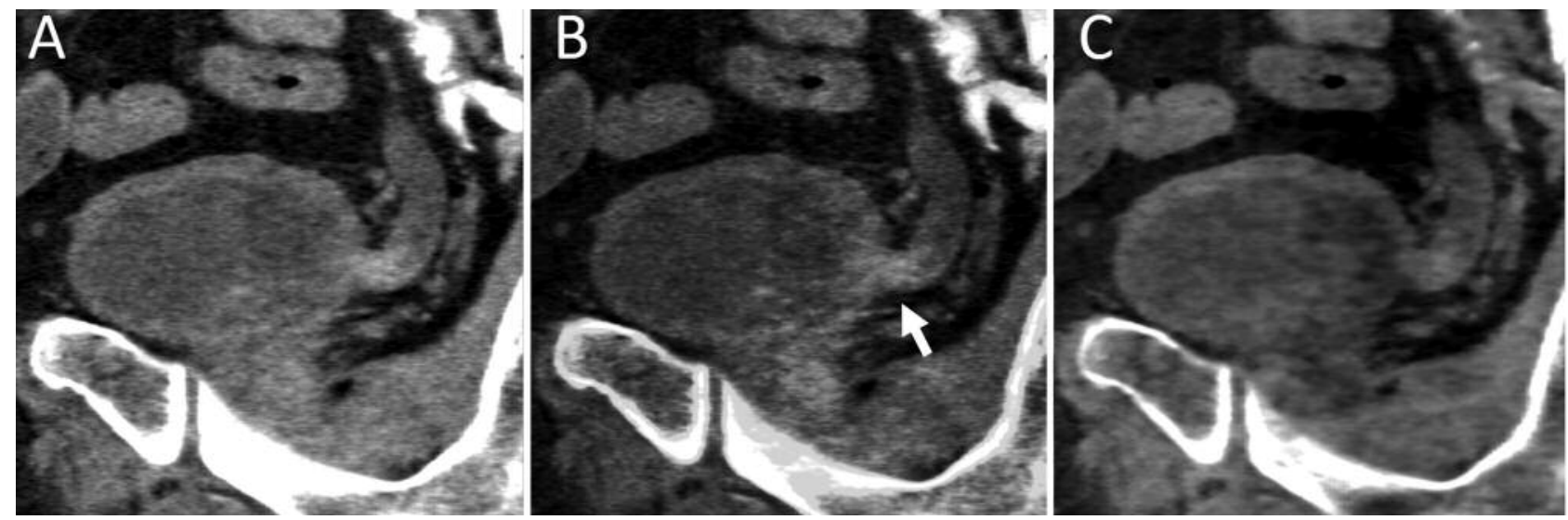

Figure 2. Very small transition cell carcinoma of the distal ureter. A: Conventional enhanced image (DECT averaged image). B: DECT iodine map showing an enhancement of tumorous tissue (arrow) and absence of enhancement in coagulated blood localized inside distal ureter. C: DECT virtual unenhanced image showing the coagula within the distal ureter and low density of tumorous tissue.

density, not only calcium containing urinary stones and ossified tissues, but also uric acid stones. Furthermore, it allows evaluation of bleeding, the high CT density of which is caused by a high content of proteins. In addition to the determination of the presence of calcium and bleeding, unenhanced imaging is used in the assessment of tissue enhancement, when unenhanced images are compared with those after the intravenous application of an iodine contrast medium. While the detection of urinary stones is classically performed by unenhanced $\mathrm{CT}$, multiphase multi-detector $\mathrm{CT}$ (MDCT) is currently the first-line method indicated in differential diagnosis of renal masses. Recent studies show that multi-detector-row CT systems can fully cover the clinical requests in the therapeutic decision process in renal tumors $(10,12)$. The algorithm of CT examination in patients suffering from hematuria firmly includes unenhanced imaging, followed by contrast enhanced MDCT, finished by excretory imaging called CT-urography $(1,2,10)$. The current role of $\mathrm{CT}$ is, therefore, firmly established in evaluation of the possible sources of bleeding into the urinary collecting system - tumors and calculi.

Implementation of DECT in the imaging algorithms provides the reduction of the needed number of scans $(3,5-7)$ with the additional improvement of data evaluation by means of the evaluation of iodine content within the tissues and by the means of chemical analysis of the urinary stones. The 
results of our study demonstrate the usability of contrastenhanced abdominal imaging such as single contrast enhanced DECT for the detection and description of tumors growing into the collecting system including discrimination of the tumorous tissue and clotted blood if virtual unenhanced imaging is used for visualization of the hemorrhage and iodine distribution map for visualization of tumorous tissue. Virtual unenhanced CT scans can be reconstructed from dual-energy CT data acquired after the previous intravenous application of the contrast medium. The correct location of the tumorous tissue could be detected using combined imaging with the virtual unenhanced images and iodine distribution maps in high accuracy of $94 \%$ (Figures 1 and 2). These results show a significant potential for the detection of TCC only from one post-contrast dual-energy scan and, therefore, the possibility to omit the conventionally performed unenhanced abdominal scan and in some cases scans in the excretory phase. Regarding the radiation dose to the patient, the current dualenergy CT scan protocol exceeded 1.2 times the dose obtained from a conventional single-energy CT protocol (13).

Dual-energy data acquisition enables the reconstruction of virtual unenhanced images and iodine distribution maps, when the density of each voxel is splitting in those derived from the iodine content and those derived from natural tissue. The corresponding algorithm is based on the markedly increased X-ray attenuation of iodine at $80 \mathrm{kV}$ compared to $140 \mathrm{kV}$, because of the significant contribution of the photoelectric effect due to the high atomic number of iodine $(3,4,14)$. Other tissues show a much less pronounced change in X-ray attenuation since they consist of elements with a lower atomic number. Therefore, it is possible to calculate, for any given voxel, how the iodine content and the tissue itself contribute to the final density $(3,4)$, and to create a map corresponding to the unenhanced enhanced densities of tissues (3-5). This virtual unenhanced image created from DECT data displays the soft tissues, kidney parenchyma, clear urine, blood or calculi.

The reduced contrast-to-noise ratio did not result in poorer differentiation of structures, but influenced in some subjectively felt worsening of the imaging quality (15-17). However, no influence on the detectability of tumor or urinary stone was found, indicating the potential of omission of the unenhanced scan from the diagnostic algorithm (6).

The CT-urography is thought to be the first method of choice in the imaging of the collecting system. Being the cause of urine outflow obstruction, the massive content of coagulated blood or tumorous tissue may result in the markedly delayed excretion of the iodine. However, in some cases the imaging in the excretory phase must be repeated, which increases dose burden to the patient. Our DECT data analysis showed, that the imaging in the excretory phase has added value in the detection of hematuria cause, only in few cases, therefore it can be omitted.
Our study indicates the potential of contrast enhanced DECT analysis of virtual unenhanced imaging to replace the generally conventional approach in urinary imaging, and therefore, to significantly reduce the overall radiation burden on the patient. Further decrease of the radiation dose could be achieved using novel approaches in data reconstruction using iterative reconstruction algorithms and using the prefiltration of $X$ rays on tube using the effect of spectral shaping by high energy tin filtration (17-20).

Limitations of our study include the relative small cohort of patients which reduces the possibility to generalize the role of single, phase iodine enhanced DECT in indication in patients with bleeding. In addition, no negative cases were included and therefore specificity cannot be reported. For these reasons, further studies on this issue are needed.

\section{Conclusion}

Based on the complex data derived from a single contrastenhanced dual-energy $\mathrm{CT}$ acquisition, it is possible to distinguish with high accuracy TCC from bleeding into the urinary collecting system in patients suspected for a urinary collecting system tumor with a significant reduction of the radiation dose.

\section{Acknowledgements}

This study was supported by the project of the Ministry of Health of the Czech Republic - Conceptual Development of Research Institutions 00669806 - FN Plzen, by the project of the Charles University Prague Progress Q39 and by the project CZ.1.05/2.1.00/03.0076 from European Regional Development Fund.

\section{References}

1 Mueller-Lisse UG, Mueller-Lisse UL, Hinterberger J, Schneede $\mathrm{P}$, Meindl $\mathrm{T}$ and Reiser MF: Multidetector-row computed tomography (MDCT) in patients with a history of previous urothelial cancer or painless macroscopic haematuria. Eur Radiol 17(11): 2794-2803, 2007.

2 Tsili AC, Efremidis SC, Kalef-Ezra J, Giannakis D, Alamanos Y, Sofikitis N and Tsampoulas C: Multi-detector row CT urography on a 16-row CT scanner in the evaluation of urothelial tumors. Eur Radiol 17(4): 1046-1054, 2007.

3 Leschka S, Stolzmann P, Baumüller S, Scheffel H, Desbiolles L, Schmid B, Marincek B and Alkadhi H: Performance of dualenergy CT with tin filter technology for the discrimination of renal cysts and enhancing masses. Acad Radiol 17(4): 526-534, 2010.

4 Graser A, Becker CR, Staehler M, Clevert DA, Macari M, Arndt N, Nikolaou K, Sommer W, Stief C, Reiser MF and Johnson TR: Single-phase dual-energy CT allows for characterization of renal masses as benign or malignant. Invest Radiol 45(7): 399-405, 2010. 
5 Graser A, Johnson TR, Hecht EM, Becker CR, Leidecker C, Staehler M, Stief CG, Hildebrandt H, Godoy MC, Finn ME, Stepansky F, Reiser MF and Macari M: Dual-energy CT in patients suspected of having renal masses: can virtual nonenhanced images replace true nonenhanced images? Radiology 252(2): 433-440, 2009.

6 Ascenti G, Mileto A, Gaeta M, Blandino A, Mazziotti S and Scribano E: Single-phase dual-energy CT urography in the evaluation of haematuria. Clin Radiol 68(2): e87-94, 2013.

7 Karlo C, Lauber A, Götti RP, Baumüller S, Stolzmann P, Scheffel H, Desbiolles L, Schmidt B, Marincek B, Alkadhi H and Leschka S: Dual-energy CT with tin filter technology for the discrimination of renal lesion proxies containing blood, protein, and contrast-agent. An experimental phantom study. Eur Radiol 21(2): 385-392, 2011.

8 Altenbernd J, Heusner TA, Ringelstein A, Ladd SC, Forsting M and Antoch G: Dual-energy-CT of hypervascular liver lesions in patients with HCC: investigation of image quality and sensitivity. Eur Radiol 21(4): 738-743, 2011.

9 Ferda J, Novak M, Mirka H, Baxa J, Ferdova E, Bednarova A, Flohr T, Schmidt B, Klotz E and Kreuzberg B: The assessment of intracranial bleeding with virtual unenhanced imaging by means of dual-energy CT angiography. Eur Radiol 19(10): 25182522, 2009.

10 Fritz GA, Schoellnast H, Deutschmann HA, Quehenberger F and Tillich M: Multiphasic multidetector-row CT (MDCT) in detection and staging of transitional cell carcinomas of the upper urinary tract. Eur Radiol 16(6): 1244-1252, 2006.

11 Hansen C, Becker CD, Montet X and Botsikas D: Diagnosis of urothelial tumors with a dedicated dual-source dual-energy MDCT protocol: Preliminary results. AJR Am J Roentgenol 202: W357-W364, 2014

12 Ferda J, Hora M, Hes O, Ferdová E and Kreuzberg B: Assessment of the kidney tumor vascular supply by two-phase MDCT-angiography. Eur J Radiol 62(2): 295-301, 2007.

13 Mileto A and Marin D: Dual-Energy Computed Tomography in Genitourinary Imaging. Radiol Clin N Am 55: 373-391, 2017.
14 Agrawal MD, Pinho DF, Kulkarni NM, Hahn PF, Guimaraes AR and Sahani DV: Oncologic applications of dual-energy CT in the abdomen. Radiographics 34(3): 589-612, 2014.

15 Sommer WH, Graser A, Becker CR, Clevert DA, Reiser MF, Nikolaou $\mathrm{K}$ and Johnson TR: Image quality of virtual noncontrast images derived from dual-energy CT angiography after endovascular aneurysm repair. J Vasc Interv Radiol 21(3): 315-321, 2010.

16 Guimarães LS, Fletcher JG, Harmsen WS, Yu L, Siddiki H, Melton Z, Huprich JE, Hough D, Hartman R and McCollough $\mathrm{CH}$ : Appropriate patient selection at abdominal dual-energy CT using $80 \mathrm{kV}$ : relationship between patient size, image noise, and image quality. Radiology 257(3): 732-742, 2010.

17 Vrtiska TJ, Takahashi N, Fletcher JG, Hartman RP, Yu L and Kawashima A: Genitourinary applications of dual-energy CT. AJR Am J Roentgenol 194(6): 1434-1442, 2010.

18 Thomas C, Krauss B, Ketelsen D, Tsiflikas I, Reimann A, Werner M, Schilling D, Hennenlotter J, Claussen CD, Schlemmer HP and Heuschmid M: Differentiation of urinary calculi with dual energy CT: effect of spectral shaping by high energy tin filtration. Invest Radiol 45(7): 393-398, 2010.

19 Apel A, Fletcher JG, Fidler JL, Hough DM, Yu L, Guimaraes LS, Bellemann ME, McCollough CH, Holmes DR 3rd and Eusemann CD: Pilot multi-reader study demonstrating potential for dose reduction in dual energy hepatic $\mathrm{CT}$ using non-linear blending of mixed kV image datasets. Eur Radiol 21(3): 644-652, 2011.

20 Primak AN, Giraldo JC, Eusemann CD, Schmidt B, Kantor B, Fletcher JG and McCollough CH: Dual-source dual-energy CT with additional tin filtration: Dose and image quality evaluation in phantoms and in vivo. AJR Am J Roentgenol 195(5): 1164$1174,2010$.
Received October 26, 2017

Revised November 11, 2017

Accepted November 13, 2017 